\title{
Accountable Care Organizations: An Integrated Model of Patient Care Objectives
}

\section{RICK PANNING}

\section{LEARNING OBJECTIVES}

1. Describe how the traditional pre-analytical, analytical, post analytical model of laboratory testing will be transformed in the Accountable Care Organization model.

2. Describe the role of the clinical laboratory in the Accountable Care Organization model.

3. Describe how transitions in the health care process (i.e. discharge, transfer, etc.) add to cost and reduce quality, and how the Accountable Care Organization model is intended to address these inadequacies.

4. Delineate the differences in the three primary Accountable Care Organization models as defined in the Affordable Care Act: Medicare Shared Savings, Advanced Payment Model and Pioneer Accountable Care Organization.

ABBREVIATIONS: ACA - Affordable Care Act; ACO - Accountable Care Organization; CMS - Centers for Medicare and Medicaid Services; CPOE - Computerized Physician Order Entry; EMR - Electronic Medical Record; GDP - Gross Domestic Product; IDS Integrated Delivery Systems; PCMH - PatientCentered Medical Home; PPACA - Patient Protection and Affordable Care Act

INDEX TERMS: Patient Protection and Affordable Care Act, Affordable Care Act, Accountable Care Organization, Clinical Laboratory, Medicare Shared Savings Program, Pioneer ACO, Advance Payment Model, Healthcare Reform, Patient Centered Medical Home, Integrated Care, Continuum of Care.

\section{Clin Lab Sci 2014;27(2):112}

Rick Panning, MBA, MLS(ASCP) ${ }^{C M}$, Chair, ASCLS Government Affairs Committee, Director of Business Development for Shared Clinical Services, Fairview Health Services, Minneapolis, $M N$
Address for Correspondence: Rick Panning, MBA, MLS(ASCP) ${ }^{C M}, 4894$ Meadow Lane, Shoreview, MN 55126, 651-280-5909, panning4@comcast.net.

\section{Introduction}

As a component of the Patient Protection and Affordable Care Act (PPACA) or Affordable Care Act (ACA), for short, Accountable Care Organizations (ACO) have an ultimate goal of improving quality and patient care outcomes through coordinated care, thereby reducing the total cost of healthcare. Healthcare reform and the ACO model will shift healthcare resource allocation from acute care to an intense focus on preventive care, wellness, chronic disease and managing transitions. Because of the aging population and the increase in chronic disease, the following statistics have a significant impact on healthcare costs in the coming years. ${ }^{1}$

Seventy eight percent of the total healthcare spend in the U.S. is attributable to chronic disease. Just $5 \%$ of patients, account for greater than $50 \%$ of the total U.S healthcare expenditures. In contrast, nearly $50 \%$ of the U.S. population accounts for just $3 \%$ of total U.S. spend. Hospitals account for $31 \%$ of the total spend while medications account for $23 \%$. The outpatient laboratory spend is approximately $3.8 \%$ of the healthcare spend in this country. As you can see from these statistics, the laboratory is not the problem, but with healthcare reform and the ACO model, laboratories can be a significant part of the solution. ${ }^{1}$

The ACO model is projected to save $\$ 4.9$ billion over the first ten years of implementation. As defined in Section 3022 of the ACA, an Accountable Care Organization is a group of providers who are "accountable for the quality, cost and overall care" of patients. ACOs are essentially partnerships between health insurers/payers, hospitals, primary care physicians and post-acute care providers (long-term care and home care) to manage all health care for its 
members. This concept, not limited to federal healthcare reform envisions multiple providers assuming joint accountability for improving the quality of care and slowing the growth of health care costs. The success of the ACO model relies on its success in incentivizing hospitals, physicians and post-acute care providers to form partnerships that facilitate coordination of care delivery across the settings of care. It is the transitions in health care that most contribute to the excessive cost and waste in the system.

ACOs are a group of providers that work together to coordinate care and share in the costs saved by reducing the cost of care. ACOs are intended to be patientcentered, involving coordination, transitions and patient involvement. It is intended that ACOs demonstrate innovation in achieving better value for the system and the patient. To support this, data must demonstrate improved healthcare delivery and outcomes. In order to achieve savings and earn incentives, the ACO must meet quality standards in five areas - patient caregiver experience, care coordination, patient safety, preventative health and elderly health/atrisk populations. From a reimbursement perspective, for chronic disease the ACO will transition from fee-forservice to a model of population management and reimbursement in the form of a bundled payment. This is a significant paradigm shift for the healthcare industry.

The incentives in the program are paid by withholding a percentage of the hospital's DRG payment for the first 5 years of the program (starting at $1 \%$ and decreasing over five years) and then rewarding with incentives starting at $1 \%$ and increasing to $2 \%$ over five years. Again, incentives are only achieved if quality measures are met and cost of care is reduced. ${ }^{2}$

\section{Medicare offers three ACO programs}

The first ACO program defined in ACA is the Medicare Shared Savings Program. It is expected that this model will improve beneficiary outcomes and increase the value of care by promoting accountability for care, requiring care coordination across the continuum and requiring investment in infrastructure and improved care processes. These ACOs will be rewarded for improving costs and improving the patient care experience.
The second ACO program included in the ACA is the Advance Payment Model. This model is designed for physician-based and rural providers who have come together voluntarily to give coordinated high quality care to the Medicare patients they serve. Through the Advance Payment ACO Model, selected participants will receive upfront and monthly payments, which they can use to make important investments in their care coordination infrastructure. The advance payments described are paid back from future savings.

The final ACO model is the Pioneer ACO model. This model is designed for health care organizations and providers that are already experienced in coordinating care for patients across care settings. It will allow these provider groups to move more rapidly from a shared savings payment model to a population-based payment model on a track consistent with, but separate from, the Medicare Shared Services Program. And it is designed to work in coordination with private payers by aligning provider incentives, which will improve quality and health outcomes for patients across the ACO, and achieve cost savings for Medicare, employers and patients. $^{3}$

Accountable Care Organizations share a number of common attributes. These include that it is a regional approach to organize and deliver care for a defined population of patients; it is a true economic partnership between physicians, hospitals, health systems and purchasers and payers; risks are shared and incentives are aligned among the players; there is a defined goal to reduce fragmentation of care and redundancy in the system; there is a focus on primary and preventive care; and finally, there needs to be a comprehensive continuum of health care services across settings and levels of care - primary care, specialists, hospitals, home care and skilled nursing. ${ }^{4}$

ACOs are one mechanism that healthcare reform is counting on to "bend the cost curve" in health care and to reduce waste and efficiency while improving the quality of care. There are many ways that one can look at the rising cost of healthcare. One view that demonstrates the dramatic increase in healthcare costs over time and also shows the impact on our overall economy is the percent of Gross Domestic Product (GDP) that healthcare composes. Gross Domestic Product is a measure of the health of a nation's 
economy. It is defined as the monetary value of all the finished goods and services produced within a country's borders in a specific time period. In Figure 1, we see over 60 years that health care costs have risen from comprising $6.2 \%$ of our GDP to a projected $19.3 \%$ in 2019. Most countries in the industrialized world have a GDP in the range of $9.5-11.5 \%$. This difference of 8 $10 \%$ adds a significant competitive disadvantage as the United States attempts to compete in a global market. In addition, although we spend more and our rate of increase is much higher, the outcomes data is not uniformly better than other industrialized countries ${ }^{2}$ (Figure 2).

The ACO model, as a component of national healthcare reform, was proposed as a demonstration project to be administered by the Centers for Medicare and Medicaid Services (CMS). Along with a bundled payment model and other initiatives that address care delivery, the ACO organizations agree to assume accountability for improving quality of care and the overall cost of care for a defined and assigned patient population of Medicare beneficiaries. The ACO will receive any savings achieved, as long as the prescribed quality benchmarks are achieved. The goal is to improve patient outcomes and quality and reduce overall cost. The ACO defined in the legislation limits the financial exposure of the providers because a fee-for-service reimbursement still exists. Other ACO models in the industry will be paid a fixed amount and are therefore responsible for financial gains and losses. ${ }^{4}$

\section{National Health Spending as a Share of GDP, 1960-2019*}

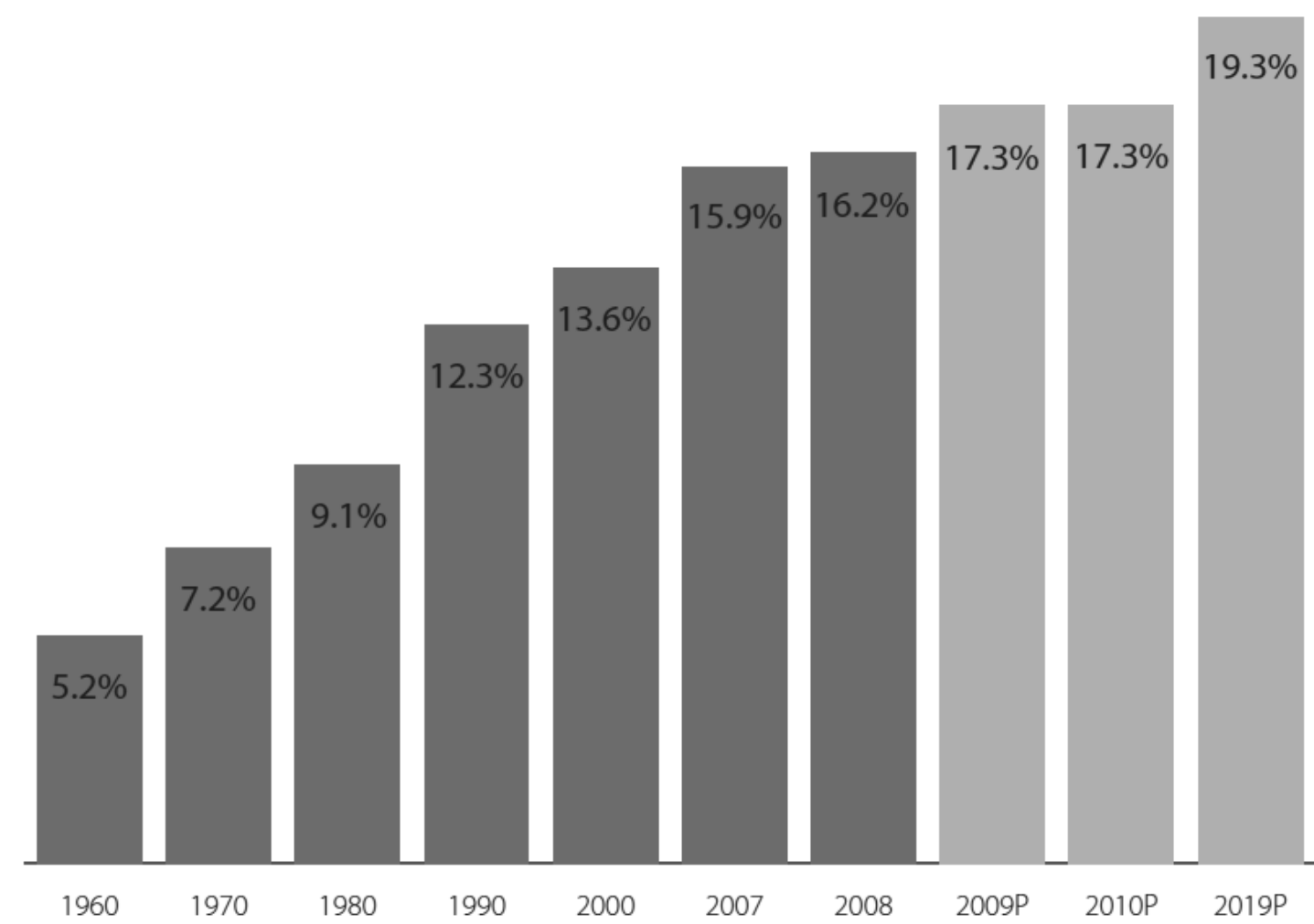

\footnotetext{
Selected rather than continuous years of data shown prior to 2007 . Years 2009 forward are CMS projections. The 2009 CMS projects reflect a 5.7 percent increase in health spending and a 1.1 percent decrease in GDP, expected to result in the largest one-year increase in history of health spending as a share of GDP; NHE's 2010 projected share of GDP reflects a 3.9 growth in NHE and 4.0 percent growth in GDP. The full projection period, 2009 to 2019, reflects CMS assumptions of an average annual increase of 4.4 percent in GDP and 6.1 percent in national health spending.
}

Figure 1. National Health Spending as a Share of GDP. 1960-2019. Source Centers for Medicare and Medicaid Services (CMS), Office of Actuary (www.cms.gov) 


\section{International Comparison of Spending on Health, 1980-2008}

\section{Average spending on health per capita (\$US PPP)}

Total expenditures on health as percent of GDP
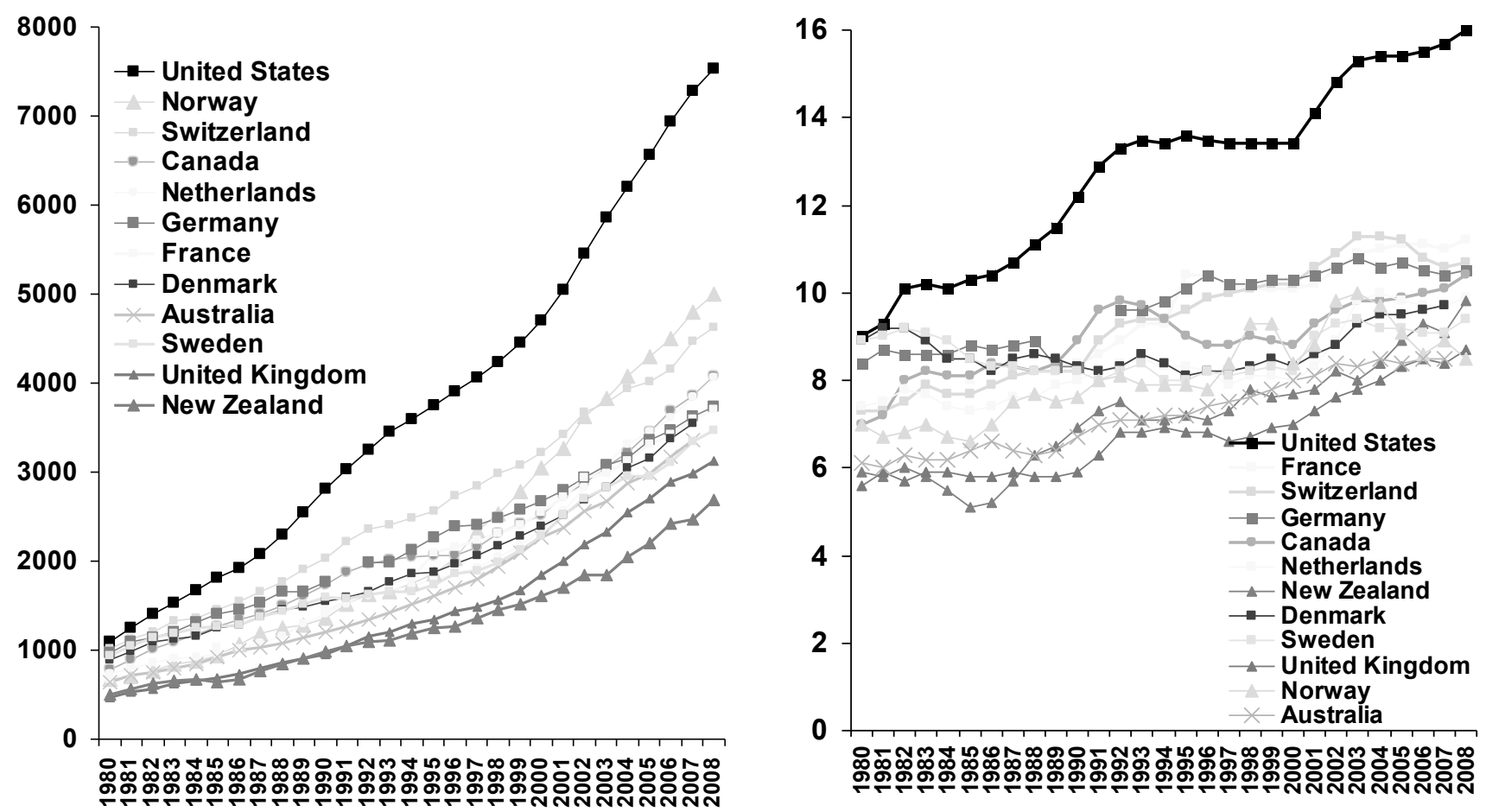

Figure 2. Trend of spending on health in industrialized countries (1980-2008). Source OECO Health Data, Oct. 2010

There are two other care delivery initiatives that need to be distinguished from ACOs. The patient-centered medical home $(\mathrm{PCMH})$ initiative emphasizes the role of the primary care physician across the care continuum and is actually complementary to the ACO. One difference is that the PMHC initiative does not offer explicit incentives and by requiring the primary care physician to take responsibility for care coordination, without the supporting resources of an ACO, that may not be possible. The Health Maintenance Organization (HMO) structure, which has existed for decades, is mainly different from an ACO due to the mechanism of payment. HMOs are typically arranged around capitation while the ACO model in healthcare reform still relies on fee for service with an additional layer of risk and incentive. That additional layer, in a "sharedsavings" model qualifies the ACO to any savings achieved. Other ACO models can be described as "bundled payment" which is negotiated for the combined ACO providers for an episode of care, which may cross provider organizations, and is therefore somewhat similar to an HMO. Where the HMO failed is that it was primarily and almost completely about cost reduction and therefore often sacrificed quality of care. One can regard the ACO model as learning from the failures of the past.

The implementation of the ACO model is intended to achieve a number of results, including less high-tech diagnostic imaging, fewer preventable emergency department visits, fewer ambulatory care-sensitive hospital admissions, fewer surgeries, fewer hospitalacquired infections and associated complications, fewer preventable readmissions (usually measured within 30 days post-discharge) and finally fewer laboratory tests and greater use of reflex testing and care protocols.

\section{Structure of an ACO}

In most ACOs the health insurer will designate a block of members or beneficiaries and the ACO will assume complete risk for the healthcare of those patients. This means that the insurer/payer will provide a bundled 
payment for each member on an annual basis to manage the entire patient health continuum. Currently there are over 400 certified ACOs in the United States, covering 25-30 million patients. There are an additional 200 ACOs expected in the coming years and the number of covered lives is expected to grow dramatically.

For an ACO to be successful it needs to focus on the following core competencies during its initial three-year commitment. $^{2,4}$ The ACO must have a defined leadership with a formal legal structure, employ enough primary care providers to treat the defined beneficiary population as defined by CMS, implement a mechanism to promote evidence-based medicine, report on quality and cost measures and coordinate care, possess an organizational culture of teamwork, develop a structure and relationship with other partners, maintain an information technology infrastructure for population management and care coordination, implement an infrastructure for monitoring, managing and reporting quality, manage financial risk, receive and distribute payments and savings, possess resources for patient education and support, spread and disseminate best practices, establish linkages and reach out to public health and community resources, and participate in regional health information exchanges to share health information in order to improve the health of the community. ${ }^{5}$

The structures, already possessed by many integrated delivery systems (IDS), may enable the formation of an ACO because the competencies and infrastructure required are likely to already be in place. One key factor is the ability to manage population health data and leadership and legal structures required. In a complementary way, many of the ambulatory care delivery models may meet a number of the required competencies including the ability to work with and coordinate with post-acute care provider organizations.

\section{Quality Benchmarks and Measures}

Since accountability to key benchmarks is an important component of the ACO, the organization must develop reliable ways to measure and report these benchmarks, which will be developed by CMS. It is important that the measures are well-defined and can be interpreted in a standardized fashion. The measures have the difficult challenge of needing to be applicable in different care settings and providers. Ultimately the benchmarks and measures will address process, outcome and patient experience. To date, the most visible benchmark has been the number of preventable readmissions within 30 days for congestive heart failure.

\section{The Laboratory's Role in Accountable Care Organizations}

What is the laboratory value proposition? Laboratory results enable preventative care, wellness and the management of chronic disease and impact a high percentage of all clinical decisions and are a significant percentage of the data in the electronic health record. Laboratory data is essential to manage coordinated care across the continuum and in managing the many patient transitions that occur in healthcare. Ultimately the laboratory is a significant enabler for quality, effective healthcare for patients.

In a white paper published by Associated Regional and University Pathologists Laboratories, the authors discuss the idea that rather than only thinking of the laboratory process in terms of the pre-analytical, analytical and post-analytical phases that we need to think more of the laboratory as an integrator of clinical data. With that paradigm, the three phases then become clinical direction - supporting and helping to define computerized physician order entry (CPOE), clinical decision support and clinical pathways, generator/distributor of clinical information and analytics - aggregation of clinical data. ${ }^{6}$ (Figure 3 )

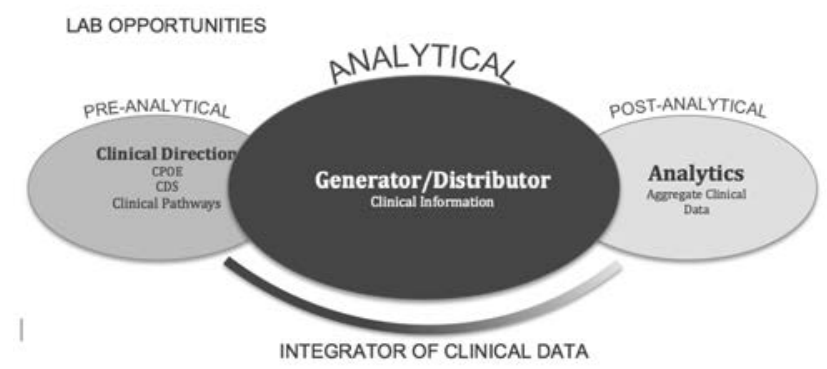

Figure 3. The role of the clinical laboratory in the Accountable Care Organization model. Source: Miles, J. and Weiss, R. The Role of Laboratory Medicine in Accountable Care Organizations. ARUP Laboratories. 2011.

With this framework, when one thinks about the needs of the primary care physician, the partnership that the 
laboratory needs to develop and maintain will involve readily available laboratory data (information systems and timeliness), clinical decision support, process improvement to assist in streamlining practice and building a strong, two-way relationship. The opportunities that the laboratory must take advantage of include developing outreach services outside of the four walls of the health system or hospital, building electronic connectivity solutions, which integrate data with the physician's electronic medical record (EMR) and assuring that operations are cost effective. One way to achieve this is through lean process improvement. Healthcare reform is about identifying and reallocating wasted resources. We need to find savings through efficiencies and operational integration. Finally, the laboratory must develop utilization management tools and guidelines and understand the role of the laboratory in the bigger picture of the overall healthcare process.

It is trite to say, but healthcare is changing and the change does not happen overnight. It takes time. Change in healthcare requires physician leadership. The laboratory's ability to develop collaborative physician relationships will always be value-added. Hospital care is expensive care and any role the laboratory can play to support getting patients out of the hospital and into the outpatient, home care or transitional and skilled nursing environment will add value to the system. Being able to provide the right information at the time of care is going to be transformational. Digital information and integration is much more powerful than paper.

In this country, a large number of laboratories are hospital-based. In order for healthcare reform and the accountable care organization to be effective, hospitals must re-engineer themselves. This will involve expanding the mission to include pre-hospital and postacute care. The focus must shift to outpatient care, while not losing the needed expertise in inpatient care processes. Hospitals are more and more becoming physician lead and must invest heavily in IT solutions, which will enable the organization to integrate information and services across the entire ACO continuum of care. On the reimbursement side, the structure of the ACO, involving the hospital, must be able to partner and develop the ability to receive and distribute bundled payments. The bottom-line is that there needs to be renewed and stepped-up focus on operational efficiencies, cost management and reduction of unnecessary care (readmissions, unnecessary treatments and testing). ${ }^{7}$

In a nutshell the value equation for the clinical laboratory needs to move from a pure fee-for-service process where value is simply reimbursement minus cost to one, under the ACO and the Medical Home, where value comes from the clinical benefit minus the cost. In the past, the role of the laboratory was to support the analytic process, assess test costs and provide the doctors with what they were asking. In the future, this needs to move to supporting the complete diagnostic process across the continuum of care, to assess the "value" of the testing we perform and to actively manage test utilization. Inconsideration of this last point, we have an extremely important facilitation and support role in a physician-led utilization management process.

Our laboratory industry needs to become more integrated in the healthcare process, much like the pharmacy industry did in the 1980s. They moved from pharmacy as a commodity which received, processed and distributed medications to one, which has a professional mindset and inter-collaboration model, developing and managing a medication formulary, optimizing individual patient medication management and educating clinicians real-time.

Similar to pharmacy, in order for the laboratory to develop a more integrated practice model, we need to do the following to add clinical value. Make sure that we listen to the "voice of the customer" and develop user-friendly test menus, detect and manage inappropriate test orders, develop a laboratory test formulary, format laboratory result screens and reports to flow the way the clinician used them, and provide diagnostic decision support. Examples that are welldescribed in the literature are at the University of Rochester and the University of Michigan. Finally, we need to make our test results easier to interpret and provide active support for test interpretation and selection. This is an enormous opportunity for clinical pathologists, medical laboratory scientists and the developing role of the clinical laboratory science doctorate.

With the ACO being a model for integrated care, the laboratory will be expected to provide and enable patient outcomes, not just individual tests. The 
laboratory will need to move to a model where income is related to the patient or the covered life and not to the volume of testing. The laboratory will be evaluated by its contribution to improving the patient care process.

In the different ACO models your laboratory can be either a "vendor" or a "member". In the vendor relationship you can add value by driving and supporting ACO analytics and performance, access some of the shared savings and gain market share as a reputable vendor. If the laboratory organization is regarded as an ACO member, one can be a part of the ACO leadership structure, and can drive ACO outcomes and performance and assure that the value that is contributed to the organization is recognized. As the laboratory leaders think about value, it is essential that the laboratory must provide accurate, reliable, timely and low cost testing. Most laboratories are already doing a good job of this. It is imperative that the laboratory provide support for appropriate clinician test selection, provide clinical test interpretation and focus the laboratory on personalized medicine. To provide value and enable high quality patient care, the laboratory must implement essential, new technologies and integrate laboratory information across the continuum - this is essential. Laboratories should specifically focus on the expected increases in care that will be provided in long term care and behavioral health and work to improve access to your services.

\section{Conclusion}

As of January 1, 2013 there were an estimated 400+ public and private ACOs in 43 states. Specifically related to Medicare and healthcare reform there were 32 Pioneer ACOs beginning in January 2012. In the Medicare Saved Savings program the number of ACOs had increased from 27 in April 2012 with an additional 89 added in July 2012 and additional 106 in January 2013 for a total of 222. If you combine all ACO models more than 400 ACOs have been certified with another 200 in process. The early consensus is that the ACO model appears to be a care delivery model which appears to be able to be a driver of both quality improvement and cost control ("bending the cost curve"). ${ }^{7}$

The formation of ACOs, healthcare reform, and Medicaid expansion will drive an overall increase in testing. ACOs will also drive a decline in hospital lab testing due to an expected significant decline in hospital census.

\section{REFERENCES}

1. Health Care Cost and Utilization Report-2012. Accessed December 30, 2013 www.healthcarecostinstitute.org.

2. Centers for Medicare and Medicaid Services (CMS). Office of the Actuary.

3. ACO Fact Sheets. Accessed December 30, 2013 www.cms. gov/Medicare/Medicare-Fee-for-Service-Payment/ACO

4. American Hospital Association Committee on Research. Accountable Care Organizations: AHA Research Synthesis Report. June 2010.

5. Health Care Reform Bill Summary: A Look at What's in the Bill. Accessed November 23, 2013 www.cbsnews.com/news/ health-care-reform-bill-a-look-at-what's-in-the-bill/

6. Miles, J. and Weiss, R. The Role of Laboratory Medicine in Accountable Care Organizations. ARUP Laboratories. 2011.

7. Leavitt Partners Center for Accountable Care Intelligence, January 2013. 